\title{
LiFi for Industrial Wireless Applications
}

\author{
Volker Jungnickel, Pablo Wilke Berenguer, Sreelal Maravanchery Mana, \\ Malte Hinrichs, Sepideh M. Kouhini, Kai Lennert Bober, Christoph Kottke \\ Fraunhofer Heinrich Hertz. Institute, Einsteinufer 37, 10587 Berlin, Germany \\ volker.jungnickel@hhi.fraunhofer.de
}

\begin{abstract}
We propose a new system concept for LiFi in industrial wireless applications. A distributed multi-user multiple-input multiple-output (MU-MIMO) architecture is used, enabling seamless mobility, reliable low-latency communications, and integration with positioning and 5G. (C) 2020 The Author(s)
\end{abstract}

OCIS codes: $060.4510,060.2330,060.2605,060.4250$

\section{Introduction}

LiFi is a short-range optical wireless communication (OWC) technology using light for mobile communications. $\mathrm{LiFi}$ is useful in indoor areas where additional wireless spectrum is needed. It does not penetrate walls and is robust against electromagnetic interference and jamming from outside the building. LiFi is based on a network of small cells, which reuses the spectrum frequently and serves only few users in each cell. LiFi is useful to complement mobile radio by offloading a significant amount of traffic to the optical spectrum. The objective of this paper is to highlight the potential of LiFi for industrial wireless applications and particularly to support reliable low-latency services, such as connecting cameras (i.e. the eyes of a mobile robot) to the artificial intelligence in the cloud. The challenge is to replace short wires by wireless links and retain mobility, while keeping latency low and reliability high. In this paper, we propose a distributed multi-user multiple-input multiple-output (MU-MIMO) architecture for LiFi in industrial applications. It supports sufficient network capacity and enables seamless mobility in large indoor areas while offering low latency and high reliability. We show how this architecture can be implemented by using distributed optical frontends (OFEs) connected via plastic optical fiber (POF) to a central unit (CU). A wideband physical layer (PHY) enables sufficient data rates $(\leq 100 \mathrm{Mbit} / \mathrm{s})$ with low power. Deterministic scheduling in space and time is applied to reuse the spectrum efficiently. Integration of LiFi with precise positioning and $5 \mathrm{G}$ promises a powerful solution to address the needs of industrial wireless applications.

\section{Initial Proof-of-Concept}

First multiple-input multiple-output (MIMO) measurements in an industrial environment [1] have been conducted using the channel sounder hardware similar to that depicted in Fig. 19. Results helped to identify two main challenges for reliable OWC in industry. The first, line-of-sight (LOS) blockage, can be mitigated by spanning a MIMO channel and using larger transmit/receive angles as illustrated in Fig. 1 $\mathrm{p}$. The overlap implies that the mobile device has free LOS to multiple OFEs and yields resilience in case a LOS should be blocked. Large angles cause the second challenge, which is reduced received signal strengths and lower signal-to-noise ratios (SNRs), consequently. The benefit of spatial diversity in the MIMO channel has been shown recently in an initial proof-ofconcept (PoC), which demonstrated a reliable real-time LiFi link in an industrial environment [2] (see Fig. 10 and the video in [3]). A practical system needs to be scaled up to cover larger areas, however. In the following, several aspects are highlighted that will need further research.
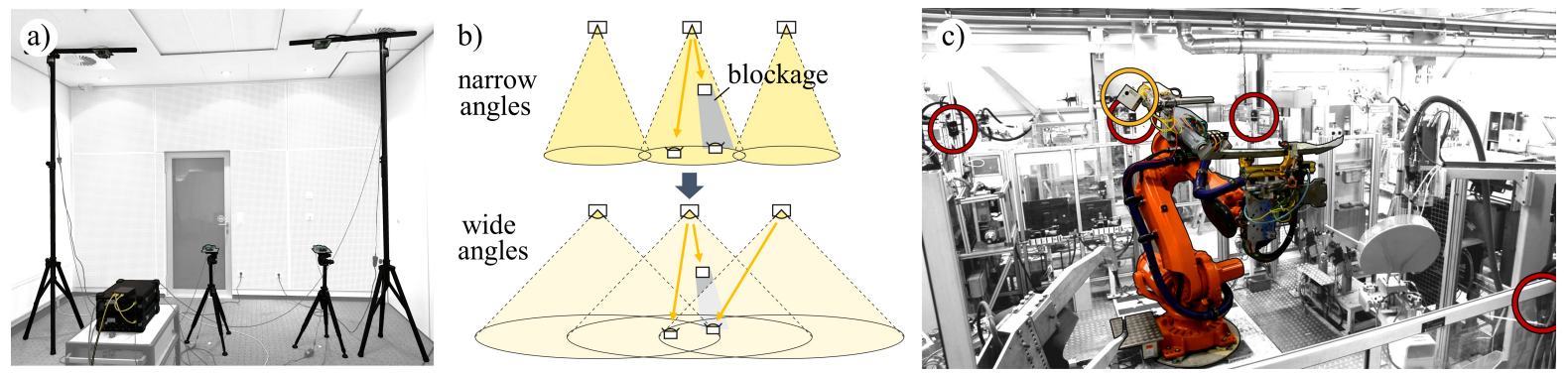

Fig. 1. a) 6x8 MIMO channel sounder. b) Multiple Tx/Rx with large transmit/receive angles mitigate the impact of LOS blockage. c) The industrial scenario used for an initial PoC. Yellow (red) circles highlight mobile (fixed) units, respectively. 


\section{Channel Modeling}

Channel modeling is needed for i) performance evaluation of different transmission schemes and ii) network planning and optimized deployment. For i), there is no statistical model yet and the common approach is ray tracing. There is an IEEE P802.11bb model for an industrial channel scenario [4], in which non-LOS signals are more relevant than usual. First mobile experiments indicated that blockage due to objects and possible rotation of the mobile robot is likely [1]. Mobile channel models are verified by measurements in realistic scenarios. For industrial applications, we have made new OFEs with wide angles and high power and use them for channel sounding. Initial measurements in an empty room are reported in [7]. For ii), as ray tracing operates in the time domain, modeling MIMO and mobility becomes very complex. Recently, a simpler technique operating in the frequency domain has been introduced [5]. Further, only the LOS and first diffuse reflections have a noticeable effect on the committed information rate (CIR). Later reflections can be approximated by the sphere model [6]. These ideas will support efficient planning of future LiFi networks and optimized deployment.

\section{Low-power PHY}

For mobile devices in industry, such as handsets, smart tools, and battery-driven sensors, energy efficiency is critical. However, many LiFi systems are not optimal in this aspect. When using orthogonal frequency-division multiplexing (OFDM), a constant bias is used around which a small-signal modulation amplitude is applied to limit nonlinear distortions. Moreover, the LED driver needs linear Class-AB amplifiers, which have limited efficiency. While it is hard to make a general comparison of the energy efficiency of OFDM and pulse-amplitude modulation (PAM), PAM can offer some advantages in practical deployments. Specifically PAM-2, or on-offkeying (OOK), has the clear advantage of an ideal peak-to-average power ratio (PAPR) for the modulated signal. The full light power can be switched on or off, enabling the use of special drivers based on Class-D amplifiers operating at high efficiency. For this reason, a pulsed modulation (PM) PHY has been developed and proposed in the IEEE P802.15.13 task group. A block diagram of the PM-PHY is depicted in Fig. 2b. 8B10B line-coding and Reed-Solomon forward error correction (FEC) are applied to header and payload data to make the signals DC-free and increase robustness. Frequency domain equalization (FDE) is used for operation at high bandwidths. Simulation results show that operation is reliable down to $10.4 \mathrm{~dB}$ and $14.4 \mathrm{~dB}$ SNR in LOS and frequency-selective industrial channels, respectively, at $200 \mathrm{MHz}$ clock rate [8]. While spectral efficiency is reduced, the increased energy efficiency makes the PM-PHY promising for energy-constrained scenarios in industrial wireless applications.

\section{Distributed multiuser MIMO MAC}

For scaling up the coverage area and the number of mobile users (MUs), a distributed MU-MIMO concept has been proposed aiming to combine spatial diversity for high reliability and spatial reuse for simultaneous support of multiple users. It follows a centralized network approach, where OFEs are distributed and cover a larger area, as depicted in Fig. $2 \mathrm{a}$. They are linked to the CU through electrical or optical links using a fronthaul technology, which can be analog or digital. While analog fronthaul is simpler, digital fronthaul is more sustainable for massive deployment. A CU coordinates the transmission and reception of all OFEs jointly to all MUs. For operating the $\mathrm{CU}$, a new medium access control (MAC) protocol was proposed in the IEEE P802.15.13 task group [9]. It controls medium access in a deterministic manner so that quality of service (QoS) is guaranteed. A dynamic time-and-space division multiple access (TSDMA) maintains spatial diversity but allows simultaneous transmissions (i.e. spatial multiplexing) where interference can be ruled out. As shown in Fig. 2k, the MAC uses a superframe structure
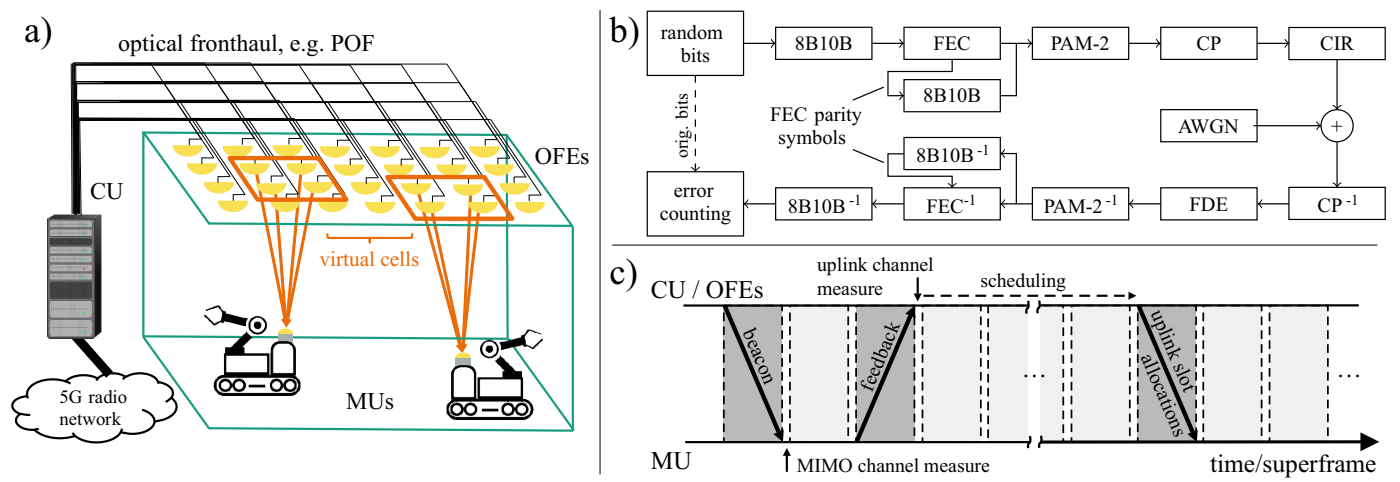

Fig. 2. a) Proposed distributed MU-MIMO system concept, b) Block diagram of PM-PHY proposed in IEEE P802.15.13, c) Essential steps of MU-MIMO MAC protocol 
to coordinate transmissions in time slots. The CU emits a periodic beacon over all OFEs. Devices measure their downlink channels from all OFEs based on orthogonal pilots which identify each OFE. Orthogonal sequences and combs are used for PM-PHY and OFDM, respectively. Each device feeds its latest channel state back to the CU. Now the CU has complete channel knowledge and broadcasts new slot allocations, scheduling MUs with mutually interfering OFE sets in different time slots and MUs with non-interfering OFE sets simultaneously.

\section{POF as Analog Fronthaul}

POF is a promising solution for transporting the signals between the CU and the distributed OFEs. Measurements in [1] indicated interference from FM radio when using twisted pair as analog fronthaul medium. Optical transmission is inherently robust against electromagnetic interference. Particularly, POF allows high data rates at low cost and easy deployment up to around $50 \mathrm{~m}$ distance. First experiments to integrate POF and OWC are reported in [10], identifying the challenge to achieve a performance mostly limited by the wireless part. Fast automatic gain control (AGC) is needed besides careful adaption between POF and OWC link to cover the required dynamic range up to $35 \mathrm{~dB}_{\mathrm{el}}$ and the channel time variance in mobile scenarios [7]. Further work indicates that the POF link should be transparent in the electrical domain, i.e. its path loss shall be fully compensated. In that case, the same fast AGC as at the mobile device can be used also at the CU, and only a static gain adaptation needs to be deployed at the distributed OFEs. Data rates can be further optimized in this way, reaching several hundreds Mbit/s, which is considered sufficient for industrial wireless applications.

\section{Realtime Positioning}

LiFi supports high bandwidth and thus can realize a next generation of indoor localization [11]. Our approach is similar to ranging in passive optical networks (PONs) and integrates the means for time-of-flight measurements to enable precise positioning. Ranging can be improved through MIMO, since it allows simultaneous synchronization and channel estimation to all visible OFEs for coarse and fine time estimation, respectively. Furthermore, a compound channel feedback can be designed as a single packet, containing the amplitudes and propagation delays of the wireless channels to all visible OFEs. This information can be updated very frequently, which allows real-time positioning for industrial applications such as position tracking for automated guided vehicles.

\section{Integration with $\mathbf{5 G}$}

5G mobile networks address new usage scenarios to attract new users and create new markets in this way. Besides enhanced mobile broadband (eMBB), which is the mainstream, the two new scenarios massive machine type communication (mMTC) and ultra-reliable and low latency communications (URLLC) are distinguished. Radio can serve larger cells more easily than OWC, so it is rational to combine $5 \mathrm{G}$ with LiFi to offload URLLC traffic and free the radio spectrum for other services. We propose LiFi as a new non-3GPP wireless access technique and to integrate it into the $5 \mathrm{G}$ core network like WiFi, by using the N3IWF interface in $5 \mathrm{G}$.

\section{Summary}

This paper has proposed a new system concept for LiFi in industrial applications. An initial proof-of-concept was provided, besides further results on low-power transmission, distributed multiuser MIMO, fronthaul using passive optical fiber, and integration of positioning and $5 \mathrm{G}$.

\section{Acknowledgements}

This work was partially supported by the EU in the H2020 projects VisIoN and ELIoT under the respective grant agreement numbers 764461 and 825651 and the German Federal Ministry of Education and Research in the project SESAM with contract number 16KIS0639K.

\section{References}

1. P. Wilke Berenguer et al., "Optical wireless MIMO experiments in an industrial environment," JSAC, 36, 1, 185-193, 2018.

2. P. Wilke Berenguer et al., "Real-Time Optical Wireless Mobile Communication with High Reliability,” JLT, 37, 6, 1628-1646, 2019.

3. https://www.youtube.com/watch?v=066jgai1Fbc.

4. https://mentor.ieee.org/802.11/dcn/18/11-18-1582-04-00bb-ieee-802-11bb-reference-channel-models-for-indoor-environments.pdf

5. H. Schulze, 'Frequency-Domain Simulation of the Indoor Optical Communication Channel," TCOM, 64, 6, 2551-2562, 2016.

6. V. Jungnickel et al., "A Physical Model of the Wireless Infrared Communication Channel," JSAC, 20, 3, 631-640, 2002.

7. Sreelal M. Mana et al., "Experiments in Non-Line-of-Sight Li-Fi Channels," Global LiFi Congress 2019, Paris, France.

8. M. Hinrichs et al., "Pulsed Modulation PHY for Power Efficient Optical Wireless Communication," ICC 2019, Shanghai, China.

9. https://mentor.ieee.org/802.15/dcn/18/15-18-0410-02-0013-mac-layer-support-for-multiple-optical-frontends.pptx

10. S. M. Kouhini et al., "Use of POF for Distributed MIMO in Li-Fi Systems," Global LIFI Congress 2019, Paris, France.

11. M. Aminikashani et al., "Indoor positioning with OFDM Visible Light Communications," CCNC 2016, Las Vegas, NV. 